\title{
Enhanced individual selection for selecting fast growing fish: the "PROSPER" method, with application on brown trout (Salmo trutta fario)
}

\author{
Bernard Chevassus $^{\mathrm{a}}$, Edwige Quillet ${ }^{\mathrm{a}}$, Francine KRIEG ${ }^{\mathrm{a}}$, \\ Marie-Gwénola HollebecQ ${ }^{\mathrm{a}}$, Muriel MAMBRINI ${ }^{\mathrm{a}}$, André FAURÉ ${ }^{\mathrm{b}}$, \\ Laurent LABBÉ ${ }^{b}$, Jean-Pierre HiseuX ${ }^{\mathrm{a}}$, Marc VANDEPUTTE ${ }^{\mathrm{a} *}$ \\ ${ }^{a}$ Laboratoire de génétique des poissons, Institut national de la recherche agronomique, \\ 78352 Jouy-en-Josas Cedex, France \\ ${ }^{\mathrm{b}}$ Station expérimentale mixte Ifremer-Inra, BP 17, 29450 Sizun, France
}

(Received 10 October 2003; accepted 30 June 2004)

\begin{abstract}
Growth rate is the main breeding goal of fish breeders, but individual selection has often shown poor responses in fish species. The PROSPER method was developed to overcome possible factors that may contribute to this low success, using (1) a variable base population and high number of breeders $(\mathrm{Ne}>100)$, (2) selection within groups with low non-genetic effects and (3) repeated growth challenges. Using calculations, we show that individual selection within groups, with appropriate management of maternal effects, can be superior to mass selection as soon as the maternal effect ratio exceeds 0.15 , when heritability is 0.25 . Practically, brown trout were selected on length at the age of one year with the PROSPER method. The genetic gain was evaluated against an unselected control line. After four generations, the mean response per generation in length at one year was $6.2 \%$ of the control mean, while the mean correlated response in weight was $21.5 \%$ of the control mean per generation. At the 4 th generation, selected fish also appeared to be leaner than control fish when compared at the same size, and the response on weight was maximal ( $\approx 130 \%$ of the control mean) between 386 and 470 days post fertilisation. This high response is promising, however, the key points of the method have to be investigated in more detail.
\end{abstract}

Salmo trutta / selective breeding / aquaculture / genetics / individual selection

\section{INTRODUCTION}

The genetic management of breeding stocks in aquaculture becomes more and more important to ensure long-term sustainable development. Growth

\footnotetext{
*Corresponding author: mvande@jouy.inra.fr
} 
rate is one of the major traits to be improved, but in many cases individual selection experiments have shown poor or even negative response in fish (e.g. [20, 27, 37]). Others were apparently more successful, but either lacked reliable control lines [8] or did not continue after the first generation [12]. Family selection seems to be more effective $[15,19,29]$. Still, efficient individual selection would be of special interest to breeders since it is simple and cheaper to set up in practical conditions. Due to the small size of fish at hatching, early individual tagging is impossible. Thus, family information can be obtained either through separate rearing of families, or by individual genotyping and parentage assignment, for example with microsatellites (e.g. $[6,11])$. Both methods are expensive, the first one because it requires large experimental facilities, and the second one because of the cost of individual genotyping (20-30€/individual).

The failure of individual selection may be explained by four main reasons:

- The low variability of the base populations: due to their high fertility, fish strains can be propagated with a limited number of breeders. This seems to be one of the main reasons for the failure of tilapia experiments [20,37] and of the carp Israeli experiment [27].

- Inbreeding may develop during the selection experiment, and have an adverse effect on growth rate $(-1.5$ to $-8 \%$ per 0.10 increase of $F$, the inbreeding coefficient $[5,30,36])$. Since high selection intensities are easy to apply in fish due to their fertility, they are especially sensitive to inbreeding during selection.

- Maternal effects may be at the origin of a large part of the phenotypic variance between individuals. Differences in hatching time may have a dramatic effect on further performance ([21] in the carp), and may occur very easily when reproduction is poorly controlled. The use of mass spawnings in some experiments $[20,27]$ may therefore explain part of their failure. Maternal effects, caused by differences in egg size, may also have an important effect on the growth performance of the individuals $[4,39]$.

- Individual selection may select the most aggressive fish, and the increase of the average aggressiveness in the group may lower their mean performance [32]. However, some results in Tilapia and medaka show that growth rate is negatively correlated with aggressiveness $[31,33,34]$. The most likely effect of social structure is the magnification of growth differences, either from genetic or environmental origin $[2,26]$.

The PROSPER process (PRocédure Optimisée de Sélection individuelle Par Épreuves Répétées $=$ enhanced individual selection procedure through recurrent challenging) was designed to overcome these potential problems in order 
to achieve an efficient individual selection in fish. We will first describe the theoretical background of PROSPER, then its application on one line of brown trout (Salmo trutta) over four generations. When the program started in 1986, brown trout was seen as an alternative to salmon in France, being able to grow in seawater under the French climate. Its main disadvantage was a low growth rate, the improvement of which was the aim of this selective breeding experiment.

\section{MATERIALS AND METHODS}

\subsection{Theoretical background of PROSPER}

Specific answers are proposed to overcome the potential limitations of the efficiency of mass selection in fish, which are reviewed in the introduction.

\section{Maintenance of genetic variability}

The base population should be chosen according to its performance for economical traits, and attention should be paid to the numbers of breeders used to found it and to propagate it. Additional information may be drawn from the variability at neutral markers, which may give indications on past bottlenecks, likely to have reduced its initial genetic variability. The numbers of broodfish used at each generation in the selection process should be high enough (in the range of $N e=100$ ) to keep inbreeding to a reasonable level.

\section{Reduction of maternal effects variance}

One possible practical source of maternal effects is the use of spawns from different days, which is the norm in production systems using natural spawning (e.g. in tilapia, seabass, seabream, in most cases). This is also a quite frequent practice in trout farms, where the spawning season for one line often lasts for more than one month. In all these cases, differences in spawning date imply differences in weights of offspring from different dams measured at the same date. Even between the offspring of females spawned on the same date, large differences in maternal effects may occur, which are mainly due to variation (assumed to be environmental) in egg size $[4,17,39]$. The method proposed for improving selection response is to undertake selection within groups of offspring from five dams with similar mean egg sizes, each group being crossed 
with a minimum of 10 sires. The rationale for this is the following: the relative efficiency of within group selection compared to individual selection [10] is:

$$
\frac{R_{w}}{R_{i}}=\frac{1-r}{\sqrt{1-t}}
$$

where $R_{w}$ is the response to within group selection, $R_{i}$ is the response to individual selection, $r$ is the correlation between breeding values of group members, and $t$ is the phenotypic correlation between group members, which can be expressed as:

$$
t=\frac{r \sigma_{A}^{2}+\frac{\sigma_{M}^{2}}{d}}{\sigma_{P}^{2}}=r h^{2}+\frac{m^{2}}{d}
$$

where $\sigma_{A}^{2}$ is the additive genetic variance, $\sigma_{M}^{2}$ is the maternal effects variance, $\sigma_{P}^{2}$ is the phenotypic variance, $d$ is the number of dams used to create the group, $h^{2}$ is the heritability and $m^{2}$ is the maternal effects ratio. Substituting (2) in (1):

$$
\frac{R_{w}}{R_{i}}=\frac{1-r}{\sqrt{1-r h^{2}-\frac{m^{2}}{d}}} .
$$

If the group is the offspring of a cross of $s$ sires with $d$ dams then:

$$
r=\frac{1}{4 s}+\frac{1}{4 d}=\frac{s+d}{4 s d}
$$

Normally, the ratio $R_{w} / R_{i}$ is lower than one. If, however, the dams within the group are chosen so that their mean egg size is equal and we can assume that there are no more maternal effects within the group (equivalent to one dam per group with respect to maternal effects, and $d$ dams per group with respect to additive variance), then equation (3) becomes:

$$
\frac{R_{w}}{R_{i}}=\frac{1-\frac{s+d}{4 s d}}{\sqrt{1-\frac{s+d}{4 s d} h^{2}-m^{2}}}
$$

Some values of $R_{w} / R_{i}$ are plotted in Figure 1, showing the superiority of the within group selection with groups from five dams and 10 sires, as soon as $m^{2}$ exceeds 0.15 when $h^{2}$ is 0.25 . It can be noted that variations in $h^{2}$ or increases in numbers of sires over 10 only marginally influence the results. Therefore, the value of 5 dams $\times 10$ sires seems appropriate. 


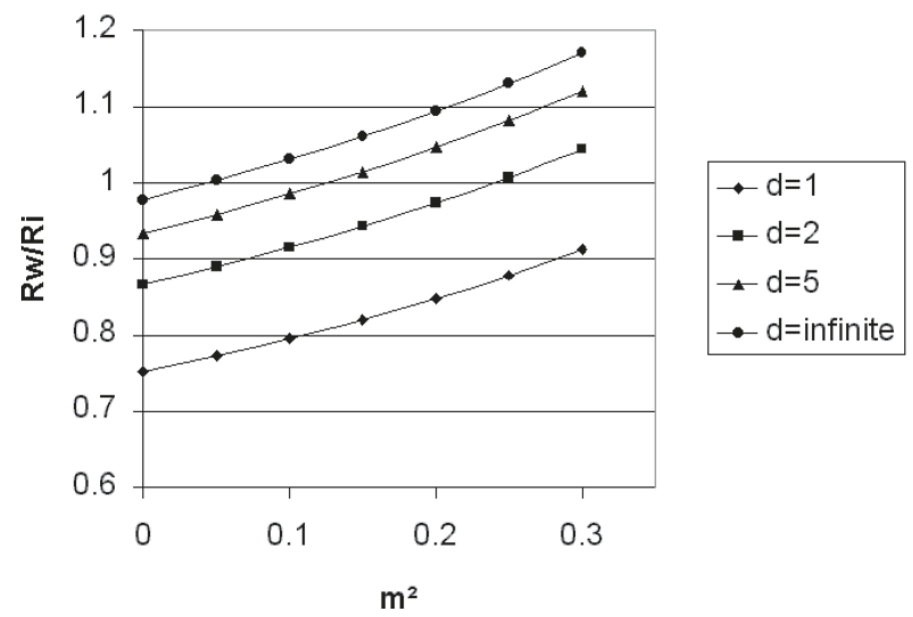

Figure 1. Relative response to within group selection $(R w)$ and mass selection $(R i)$, with groups from 10 sires and $d$ dams, $h^{2}=0.25$, for different values of the maternal effects ratio $\mathrm{m}^{2}$, under the hypothesis that maternal effects can be constrained to zero within groups.

The value of $m^{2}=0.15$ may seem high, since many studies show that the initial heterogeneity in performance between offspring of different dams progressively vanishes $[4,13,24,25]$. However, when they are reared in competition from hatching, the differences may remain [1]. When fish are first reared separately then mixed, common environmental effects (whatever their origin, maternal or environmental) may disappear [9] or not [22]. Although there is no literature estimating the maternal effects in large fish which were reared together from hatching, in brown trout, $\mathrm{m}^{2}$ on weight is as high as 0.68 at the swim-up stage [39]. There is also a substantial persistence of the initial environmental differences when fish are mixed from hatching: a $1 \%$ difference in eyed egg weight results in a $0.5 \%$ difference in weight in 3-month-old rainbow trout [1]. In another experiment on the same species, we showed that a $64 \%$ difference in eyed egg weight between the progenies of two dams, crossed with the same sires, resulted in a $34 \%$ difference in weight at 17 months of age (Dupont-Nivet, unpublished results). Proper estimation of $m^{2}$ in mixed families would require genotyping of a mixed family structure, since tagging of newborn larvae is impossible. The published data in salmonids, however, provide no $m^{2}$ estimates, either because there are not enough dams $\left(2 \circ \times 460^{7}\right.$ in [6]) to estimate the $m^{2}$, or because there are not enough sires ( 2 neomales $\times 48$ 우 in [11]) to properly separate the maternal and additive effects. However, in these two studies with rainbow trout, around $400 \mathrm{~g}$ mean weight, the estimated 
heritability of length is $0.05-0.18$ in [6], where the additive variance is estimated mainly from between sires variance, and 0.52-0.66 in [11], where it is estimated mainly from between dams variance. Although the populations and rearing conditions are different, this leaves room for significant maternal effects. Thus, the hypothesis of high values of $m^{2}$ in mixed families of salmonids seems realistic, although not formally proven.

\section{Recurrent challenges}

Even when initial environmental variability within each group has been reduced as above, phenotypic variability of growth performance, which appears soon after the fish start feeding, may still include uncontrolled environmental effects. Whatever the origin of their superiority, the largest animals tend to maintain their position in the distribution, which may hinder the expression of high growth potential in other animals $[3,14]$.

Our hypothesis was that recurrent challenges should reduce this, although it is true that one could state the exact opposite, considering that repeatedly combining fish with a similar size to a common tank, may lead to a situation in which only the really aggressive fish obtain the highest body weights. Ideally, the growth rate of the groups should be managed with feeding level and density so that all groups (although issued from different egg sizes and possibly different fertilisation dates) should reach the same mean size at 4-5 months post hatching (around $3 \mathrm{~g}$ ). All animals from the different groups are then subjected to the same challenge: they are distributed in 3 size classes, using the same truncation points for all groups (Fig. 2). Animals in the "Small" size class (approx. 50\%) are discarded, and two new groups are constituted with the "Large" and "Medium" size classes. In practice, at the time of the first challenge, differences between group means may remain, but are expected to be of purely environmental origin. As the PROSPER design implies within group selection, the means of the groups should be very close to allow the use of the same truncation points. If they are not close enough, the groups are distributed among several clusters of groups with close mean size, within which the same truncation points are applied. The management of the groups issued from the different clusters is then adapted to allow convergence in mean weight, for further merging (see the practical schemes in Section 2.1). The sorted groups (Large, Medium) have a low phenotypic variance but are assumed to have a high genetic variance. Within each cluster, the "Medium" group and the "Large" group are reared under the same density and feeding conditions (which may differ between clusters), and after a growing period 


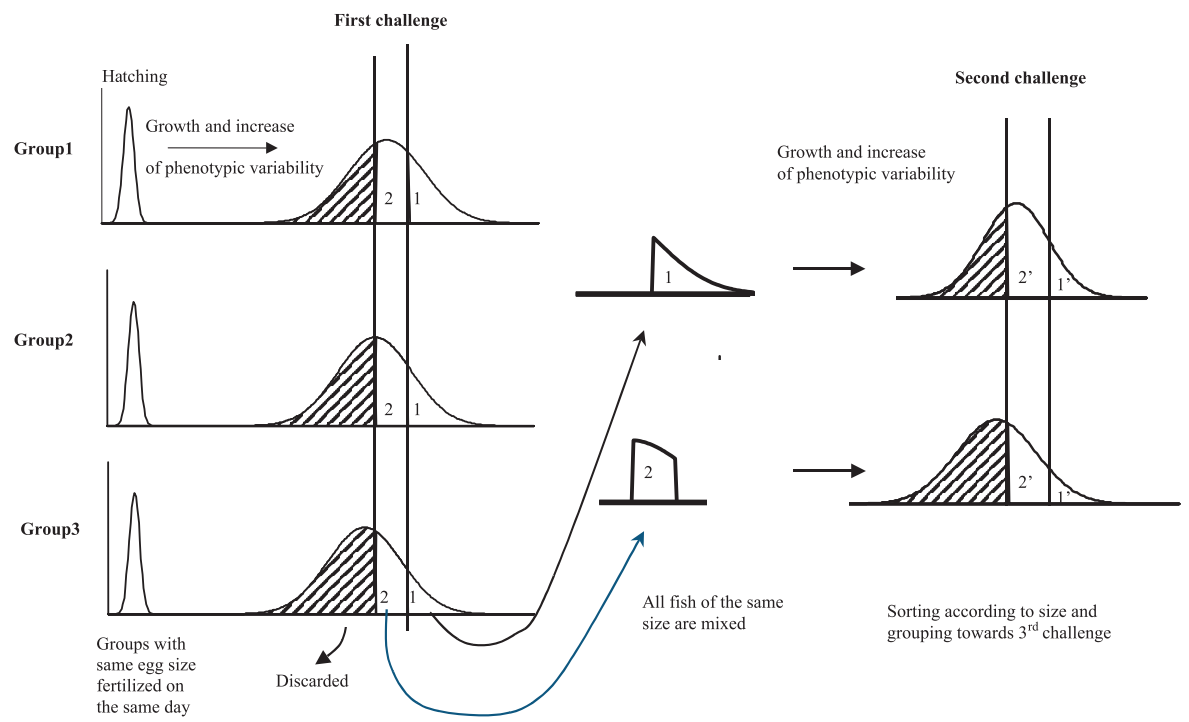

Figure 2. Principle of recurrent growth challenges in the PROSPER individual selection method.

allowing the re-expansion of phenotypic variability, the animals in both groups are re-subjected to the same type of challenge. However, at this time, the difference between group means (within cluster) is expected to be mainly of genetic origin. These challenges are to be repeated several times until a reasonable global selection pressure is achieved (around 5 to $2 \%$ ).

\subsection{Application of PROSPER to the selective breeding of brown trout}

\section{Base population}

The base population used in this experiment (NL) came from a commercial fish farm in Normandy, and was chosen among eight European domesticated and wild populations. This population exhibited a high growth rate in fresh and seawater [7], as well as a high allozyme heterozygosity [23] which was considered as a good indicator of the absence of severe population bottleneck in its history.

\section{Selection process}

The selection process followed the principles outlined before. Fork length was chosen as a selection criterion, because it is (1) highly correlated with 
weight (which remains the trait of economical interest) and (2) easy to measure on large numbers of animals under field conditions. Typically, in midNovember, two hundred 3-year-old fish were sorted as maturing females, fluent males and immature fish. Every 10 days, spawns were collected from ovulated females, and the mean egg weight of each spawn was estimated by weighing 200 eggs. Three pools of eggs from about 5 females with similar mean egg weight were constituted (1000 eggs/female), with each pool being fertilised with the same pool of sperm from 15 males. This procedure was repeated four times with different males at 10 day intervals, achieving the constitution of 12 groups, representing altogether around 60 females and 60 males. These groups were equalised to 600 fish/group and reared separately until five months post-hatching. At that time, the first selection challenge was applied. In each group, the fish were measured, the smallest 400 fish were discarded, and 100 large and 100 medium fish were kept. The "medium" and "large" groups issued from the former 12 groups which were the closest in mean size were merged two by two. Thus, 12 groups of 200 fish (6 large and 6 medium) were available after the first challenge. The fish were grown for 4 months before the second challenge. At that time, the groups were distributed into two clusters containing 3 "large" groups, as close as possible in mean size and the corresponding 3 "medium" groups. All groups within a cluster were subjected to the same thresholds. The smallest 600 were eliminated, 300 "large" and 300"medium" fish were kept within each cluster. They were grown for 4 to 6 months before the third challenge, where all 4 groups were subjected to the same selection threshold (i.e. same minimum fork length), producing one group of 300 fish. At 2 years of age, there sometimes was a fourth challenge where only the largest 200 fish were kept as future breeders. This was adapted to the numbers of breeders and rearing conditions at each generation (details in Tab. I).

Initially, the NL line was maintained at the Inra freshwater fish culture facility of Gournay sur Aronde (Oise, France), with some fish transferred to seawater for the first two generations, in order to select on both freshwater and seawater growth performance. This was stopped after the second generation due to spawning problems of seawater reared females. The NL line was then maintained in the Inra-Ifremer joint experimental freshwater farm in Sizun (Finistère, France).

In the first generation, females were separated from males from the 4th challenge in order to lower the selection pressure on them, since the sexual dimorphism in favour of males tended to increase it. In the subsequent generation, this was not done any more and then the effective selection pressure was higher 
Table I. The PROSPER selection process in brown trout.

\begin{tabular}{|c|c|c|c|c|c|c|c|c|c|c|c|c|}
\hline \multirow[b]{2}{*}{$\begin{array}{l}\text { Generation } \\
\text { (date fertil.) }\end{array}$} & \multirow[b]{2}{*}{$\mathrm{Nd}$} & \multirow[b]{2}{*}{ Ns } & \multicolumn{10}{|l|}{ Challenges } \\
\hline & & & $\begin{array}{l}\text { Challenge } \\
\text { number }\end{array}$ & $\begin{array}{l}\text { Age } \\
\text { (days PF) }\end{array}$ & Survival & $\begin{array}{l}\text { Mean } \\
\text { weight (g) }\end{array}$ & $\begin{array}{l}\text { Groups } \\
\text { before }\end{array}$ & $\begin{array}{l}\text { Groups } \\
\text { after }\end{array}$ & $\begin{array}{l}\% \\
\text { selected }\end{array}$ & $\begin{array}{l}\text { \% selected } \\
\text { (cumul.) }\end{array}$ & Sex & Used for \\
\hline SEL1 & 54 & 56 & 1 & 172 & $88 \%$ & 2.7 & 6 & 4 & $31.9 \%$ & $31.9 \%$ & & \\
\hline \multirow[t]{8}{*}{ Nov. 86} & & & 2 & 264 & $99 \%$ & 24 & 4 & 2 & $64.0 \%$ & $20.4 \%$ & & \\
\hline & & & 3 & 503 & $94 \%$ & 221 & 2 & 1 & $59.2 \%$ & $12.1 \%$ & & \\
\hline & & & 4 & 713 & $98 \%$ & 767 & 1 & 1 & $100.0 \%$ & $12.1 \%$ & $\mathrm{I}+\mathrm{F}$ & \\
\hline & & & & & & & & & $52.3 \%$ & $6.3 \%$ & M & \\
\hline & & & 5 & 860 & $96 \%$ & N/A & 1 & 1 & $60.0 \%$ & $7.3 \%$ & $\mathrm{I}+\mathrm{F}$ & SEL2 \\
\hline & & & & & & & & & $71.1 \%$ & $4.5 \%$ & M & SEL2 \\
\hline & & & $3 S$ & 532 & $38 \%$ & 268 & 1 & 1 & $65.9 \%$ & $13.5 \%$ & & \\
\hline & & & $4 \mathrm{~S}$ & 773 & $85 \%$ & 2810 & 1 & 1 & $81.0 \%$ & $10.9 \%$ & & SEL2 \\
\hline SEL2 & 63 & 84 & 1 & 199 & $86 \%$ & 9.9 & 14 & 13 & $24.8 \%$ & $24.8 \%$ & & SEL3 \\
\hline \multirow[t]{2}{*}{ Nov. 89} & & & $2 \mathrm{~S}$ & 466 & $93 \%$ & 228 & 13 & 6 & $26.5 \%$ & $6.6 \%$ & & \\
\hline & & & $3 \mathrm{~S}$ & 828 & $\mathrm{~N} / \mathrm{A}$ & $\mathrm{N} / \mathrm{A}$ & 6 & 1 & $61.3 \%$ & $4.0 \%$ & & SEL3 \\
\hline SEL3 & 43 & 72 & 1 & 196 & $94 \%$ & 10.0 & 10 & 9 & $13.4 \%$ & $13.4 \%$ & & \\
\hline \multirow[t]{2}{*}{ Nov. 92} & & & 2 & 327 & $100 \%$ & 106.3 & 9 & 2 & $40.0 \%$ & $5.4 \%$ & & \\
\hline & & & 3 & 499 & $100 \%$ & 554 & 2 & 1 & $51.5 \%$ & $2.8 \%$ & & SEL4 \\
\hline SEL4 & 56 & 55 & 1 & 186 & $76 \%$ & 9.08 & 7 & 2 & $34.4 \%$ & $34.4 \%$ & & \\
\hline \multirow[t]{2}{*}{ Nov. 95} & & & 2 & 445 & $92 \%$ & 198 & 2 & 2 & $29.9 \%$ & $10.3 \%$ & & \\
\hline & & & 3 & 551 & $91 \%$ & 456.5 & 2 & 1 & $10.5 \%$ & $1.1 \%$ & & \\
\hline
\end{tabular}

Nd: number of dams; Ns: number of sires; Age days PF: age in days post-fertilisation; N/A: not available. "Sex" indicates groups subjected to differential selection pressures according to pheonotypic sex $(\mathrm{M}=$ male, $\mathrm{F}=$ female, $\mathrm{I}=$ immature). $\mathrm{S}=$ challenges occurring in seawater, SEL $g$ : $g$ th generation of selection. 
on females than on males. The overall selection pressure was $8.3 \%$ in generation $1,9.7 \%$ in generation $2,2.8 \%$ in generation 3 and $1.2 \%$ in generation 4 .

One random-bred control line was derived from the same base population as the selected line, and propagated with 34-54 females and 46-57 males at each generation. The control line will be referred to as $\mathrm{CON} g$, and the selected line as SEL $g, g$ being the number of generations of selective breeding (or random mating for the control).

\section{Estimation of the response to selection}

The response to selection was estimated at each generation using contemporary comparisons of offspring from the selected and control line, in replicated tanks. In some cases the response was estimated through crossing of a selected or control line to another line of brown trout available on the fish farm, known as the synthetic line (SY), which was founded between 1979 and 1986 from eight different Atlantic populations of brown trout. The SY line was part of another experiment, and was used as a tester in the 2 nd and 3rd generation to save space in the experimental farm. The use of this line as a male or female tester only allowed to measure half of the genetic gain, so the observed contrast was multiplied by two to estimate the selection response. Possible heterosis cannot be ruled out, but the contrast between CON*SY and SEL*SY should not suffer from it, since both CON and SEL are derived from the same base population. The details of the comparisons used are given in Table II, in the Results section. The fish were fed ad libitum. Selection response was measured at 1 year (328 to 349 days post fertilisation) in all response estimation experiments. In each replicate (2-4 per line), 50 to 115 randomly sampled fish were weighed individually (nearest $0.1 \mathrm{~g}$ ) and measured (fork length, nearest $\mathrm{mm}$ ) - see details in Table II.

\section{Correlated response on fish shape}

The last selection response experiment occurred in the fourth generation of selection. Offspring from selected and control fish were reared each in two replicate tanks, and 100 fish were measured and weighed in each tank at 339 days post fertilisation. The Fulton condition coefficient $K$ was calculated for each fish $\left(K=10^{5} \times W \cdot L^{-3}\right.$, with $W$ the individual weight in $\mathrm{g}$ and $L$ the individual length in $\mathrm{mm}$ ). 
Table II. Estimation of selection response in brown trout on weight and length at 1 year. SEL $x$ is the selected line ( $x$ selective breeding generations), $\mathrm{CON} y$ is the control line ( $y$ random mating generations), $\mathrm{SY}$ is a synthetic line.

\begin{tabular}{|c|c|c|c|c|}
\hline & \multicolumn{4}{|c|}{ Generation of selection } \\
\hline & 1 & 2 & 3 & 4 \\
\hline Selected line & $\sigma^{\top}$ SEL1*oSEL1 & $\mathrm{O}^{7} \mathrm{SEL} 2 * \mathrm{q} \mathrm{SY}$ & $0^{7} \mathrm{SY} *$ o SEL3 & $\sigma^{\top}$ SEL $4 b *$ * SEL4b \\
\hline $\mathrm{Nb} \sigma^{x} / q$ used & $16 / 18$ & $20 / 7$ & $45 / 47$ & $11 / 34$ \\
\hline Control line & $\sigma^{\top} \mathrm{CON} 1 *_{+} \mathrm{CON} 1$ & $\sigma^{\top} \mathrm{CON} 2 * o \mathrm{SY}$ & $\sigma^{7} \mathrm{SY} \mathrm{YCON}^{*}$ & $\sigma^{\top} \mathrm{CON} 4 *{ }_{+} \mathrm{CON} 4$ \\
\hline $\mathrm{Nb} \sigma^{\top} / \mathrm{q}$ used & $22 / 18$ & $20 / 7$ & $45 / 18$ & $37 / 27$ \\
\hline Age at measurement (d.p.f.) & 349 & 338 & 328 & 345 \\
\hline Number of replicate tanks/genotype & 2 & 2 & 4 & 2 \\
\hline Fish measured/tank & 50 & 50 & 115 & 100 \\
\hline Selected length $(\mathrm{mm} \pm \mathrm{SD})$ & $206 \pm 15$ & $201 \pm 17$ & $197 \pm 20$ & $225 \pm 14$ \\
\hline Control length $(\mathrm{mm} \pm \mathrm{SD})$ & $193 \pm 16$ & $188 \pm 13$ & $177 \pm 17$ & $180 \pm 17$ \\
\hline 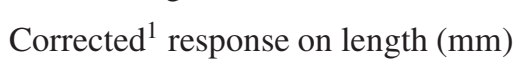 & 13 & 26 & 40 & 45 \\
\hline Selected weight $(\mathrm{g} \pm \mathrm{SD})$ & $115.3 \pm 28.1$ & $105.3 \pm 30.2$ & $101.0 \pm 30.8$ & $148.8 \pm 32.7$ \\
\hline Control weight $(\mathrm{g} \pm \mathrm{SD})$ & $95.6 \pm 25.1$ & $82.8 \pm 19.4$ & $71.9 \pm 22.1$ & $79.9 \pm 27.0$ \\
\hline Corrected $^{1}$ response on weight $(\mathrm{g})$ & 19.7 & 45.0 & 58.2 & 68.9 \\
\hline
\end{tabular}

${ }^{1}$ Taking into account the fact that only half of the response is estimated in generations 2 and 3 by the contrast between the "Selected" and the "Control" genotype, since they are crossed on a synthetic line. 
An analysis of covariance was conducted (SAS-Glm), first checking for homogeneity of slopes within replicates, then using the following model:

$$
Y_{i j k}=\mu+a \cdot L W_{i j k}+G_{i}+R_{j(i)}+e_{i j k}
$$

where $Y_{i j k}$ is the condition coefficient of the $k$ th fish in the $j$ th replicate of the $i$ th line, $\mu$ is the population mean, $L W_{i j k}$ is the natural logarithm of the weight of the fish, $a$ is the regression coefficient of $Y_{i j k}$ on $L W_{i j k}, G_{i}$ is the fixed effect of the line ( $i=1,2$; selected or control), $R_{j(i)}$ is the random effect of the $j$ th replicate $(j=1,2)$ within the $i$ th line, and $e_{i j k}$ is the random residual.

\section{Variation over the life cycle of the correlated response on weight}

In the last selection response experiment (4th generation of selection), 50 fish per tank were weighed at regular intervals from 89 to 588 days post fertilisation. At each time, the selection response was estimated as the percent weight superiority of the selected line over the control line, using the formula $R_{\%}=100\left(\frac{W s}{W c}-1\right)$, with $W s$ and $W c$ the mean weight of offspring from the selected and control lines, respectively.

\section{RESULTS}

\section{Response to selection at 1 year}

The results are given in Table II and plotted in Figure 3. The response on length at one year, estimated at the 4 th generation, was $24.6 \%$ of the control mean $(6.2 \%$ per generation). The correlated response on weight was $86 \%$ of the control mean $(21.5 \%$ per generation). The increase in response from the 3 rd to the 4 th generation was lower than in the preceding generations.

\section{Correlated response on fish shape}

The slopes of the regression of $K$ on $\ln$ (weight) were homogeneous among lines and replicates, so standard analysis of covariance could be used. It showed that:

- the regression of $K$ on $\ln$ (weight) was highly significant $(P<0.0001$, $\mathrm{a}=0.172$ ), demonstrating a positive correlation between weight and condition factor; 


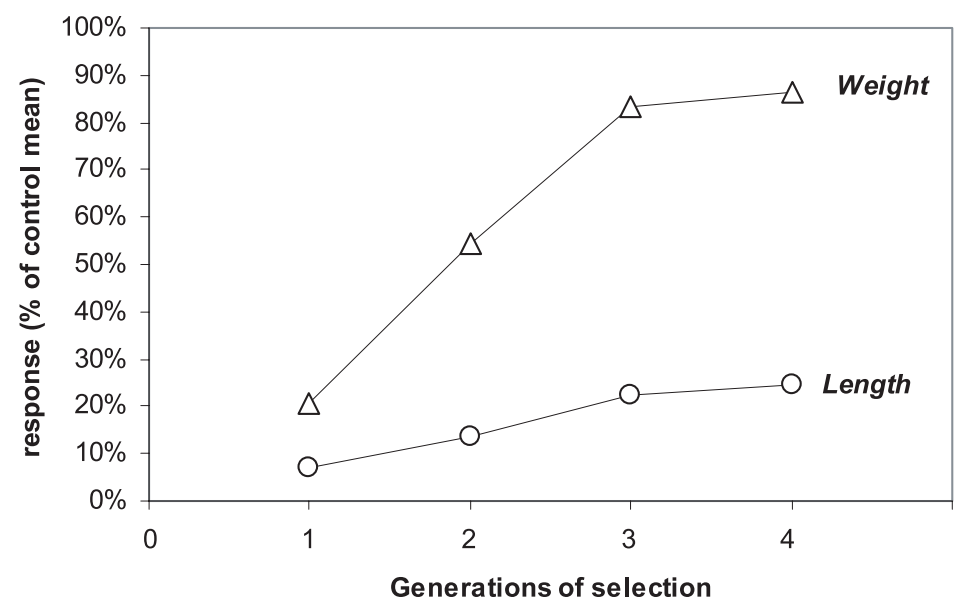

Figure 3. Selection response (in percent of the control mean) for length and weight at one year in brown trout, over four generations of PROSPER selection.

- the effect of line on $K$ was significant $(P<0.05)$, with least squares means of $K( \pm$ S.E. $)$ of $1.23 \pm 0.01$ for selected fish and $1.36 \pm 0.01$ for control fish. The same model without log-weight as a covariate gave no significant effect of line on $\mathrm{K}(P>0.6)$, with least squares means of $1.28 \pm 0.01$ for selected fish and $1.31 \pm 0.01$ for the control.

\section{Variation over the life cycle of the correlated response on weight}

The growth of the selected and control line as well as the selection response are plotted in Figure 4. The selection response starts from 4\% at 89 days, then grows until 386 days, when it stabilises around $130 \%$, before decreasing to $94 \%$ at 588 days.

\section{DISCUSSION}

This experiment yielded a large response to selection. As a reference, figures obtained in other salmonid breeding programmes are usually lower: $13 \%$ and $14.4 \%$ per generation on weight in rainbow trout and salmon (respectively) in Norway [16] using combined selection, $12.5 \%$ per generation on weight of Atlantic salmon in Canada [29] with the same procedure, and $10.1 \%$ per generation on coho salmon in Canada [19] with family selection.

However, since we did not experimentally compare it to any other method, it cannot be stated that these good results are specific to the method rather than to 


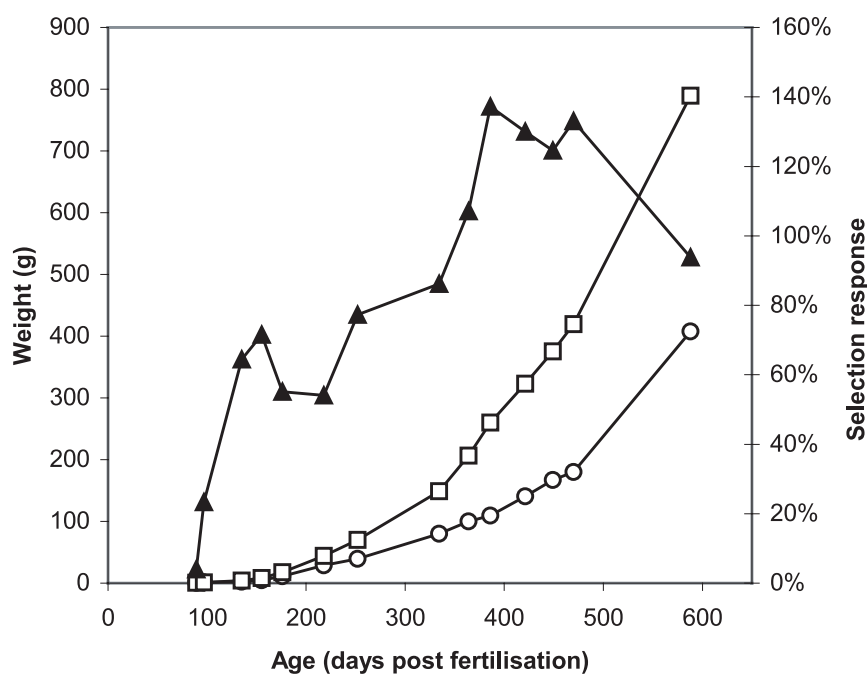

Figure 4. Establishment of the selection response for weight in the first two years in the 4 th generation of selection offspring in brown trout. $\square$ selected line, o control line, $\Delta$ selection response $(\%)$.

the stock. Moreover, family and combined selection also often include inbreeding control and selection on traits other than growth, limiting the potential gain on this last trait, which was not the case in our experiment.

Since our response is estimated as a contrast between the offspring of a random bred control and the offspring of the selected line, one possible reason for the large response would be a negative trend on the genetic value of the control, caused by inbreeding or random drift. This could be partly supported by the values of the control at each generation (Tab. II), which may however also be due to between years variation which may be very high in fish, due to their sensitivity to the environment. The variations observed may also be partly explained by the age at which the fish are measured, which varied among experiments.

The two response experiments (generations 2 and 3) that use the synthetic line as a tester tend to show higher relative responses than the experiments using pure selected and control lines. As noted before, heterosis may exist but should be the same for both CON*SY and SEL*SY crosses, since CON and SEL are derived from the same base population. However, this type of cross should also give a higher relative value to an inbred line, compared with its value as a pure line. It is well known that selection tends to increase inbreeding, and that inbreeding depression on growth exists in fish [5,30,36]. If this was the case here, this would tend to increase the contrast between SEL and 
CON when crossed with another population (the SY line). This crossing effect (which should be present in the 3rd but not in the 4th generation response estimate) may explain the small apparent increase between the 3rd and the 4th generation, despite the high selection pressure applied in the 4th generation.

The response we observed on length would imply a high heritability of this trait, in the range of $0.3-0.5$. The range of estimated heritability values for length in salmonids is large: $0.05-0.18$ in rainbow trout at 16 months [6], 0.14 at 12 months in Arctic char [28], 0.32 at first winter in Chinook salmon [40], 0.36 at 13 months in coho salmon [35], 0.53 at 215 days in rainbow trout [18]. This large variation between estimates may be due to the characteristics of the species or strains, but also to their management, as outlined in the Materials and Methods. For example, it appears that the heritability of length in rainbow trout seems much larger (0.18 compared to 0.05 ) in offspring from sorted eggs than in offspring from dams with very different egg sizes [6]. This supports the hypothesis that adequate management of maternal effects may increase the response to selection.

The selection process was based on length, which is not an economic trait per se but which is much faster and reliable to measure than weight in outdoor conditions. The correlated response on weight was very high, which may originate from a high genetic correlation between length and weight, a high heritability of weight at one year of age, and of course the high phenotypic coefficient of variation of weight when compared to that of length. A larger heritability for weight than for length is, however, not supported by the literature, since all the references cited before for length around 1 year display a similar or lower estimate for weight at the same age.

The selection on length, although it increased the weight of the selected line, did not increase the condition factor at a given age, and even tended to produce leaner fish (if compared at the same size). This demonstrates a probable negative genetic correlation between length and condition factor. This type of negative correlation has been seen at 1 year in Arctic char $(-0.17,[28])$ and in carp $(-0.38,[38])$.

The shape of the response curve in the first two years of growth, as observed in offspring from the 4th generation, is interesting. It starts quite low (4\%) at 100 days post fertilisation, and grows quickly to 50-60\% around 150-200 days post fertilisation (corresponding to the usual time of the first challenge), then it grows regularly until it stabilises at 130\% between 386 and 470 days (corresponding to the time where most of the selection pressure has been applied), and then decreases. This shows that the genetic correlation of growth rate over the different periods is lower than unity (especially for growth 
before the first challenge and after the last one). Clearly, the response is maximal at ages around the last challenge, so later challenges may in theory be useful for increasing the growth of large fish, although they may be perturbed by the sexual maturity which modifies the relative growth rates of maturing males, females and immature fish.

We have shown that the "PROSPER" selection scheme can in theory be more efficient than simple mass selection, and we demonstrated in practice that it could yield good results in brown trout over four generations. However, if the overall process is efficient, we still do not know whether it is more efficient than simple mass selection, and whether its main features (constitution of fertilisation groups, recurrent challenges) provide significant improvement or not, compared to mass selection. Further investigations, especially in the field of competitive interactions, and control of maternal effects and inbreeding are therefore needed.

\section{ACKNOWLEDGEMENTS}

The technical staff of the experimental fish farms in Gournay sur Aronde, Sizun and Camaret are warmly thanked for their cautious and rigorous practical management of the experiment for over more than 10 years. We also thank Dr. Jean-Marie Blanc for his helpful assistance, and an anonymous reviewer for constructive comments. This experiment was jointly funded by Inra and Ifremer.

\section{REFERENCES}

[1] Blanc J.M., Effects of egg size differences on juvenile weight between and within lots in rainbow trout Oncorhynchus mykiss, J. World Aquacult. Soc. 33 (2003) 278-286.

[2] Blanc J.M., Chevassus B., Poisson H., Utilisation de mutants « golden » comme témoins intra-lots pour le testage des performances de croissance chez la truite arc-en-ciel, Cybium 7 (1983) 93-103.

[3] Brown M.E., The growth of brown trout (Salmo trutta L.). I. factors influencing the growth of trout fry, J. Exp. Zool. 22 (1946) 118-130.

[4] Chevassus B., Variabilité et héritabilité des performances de croissance chez la truite arc-en-ciel (Salmo gairdneri Richardson), Ann. Génét. Sél. Anim. 8 (1976) 273-283.

[5] Chevassus B., Aspects génétiques de la constitution de populations d'élevage destinées au repeuplement, Bull. Fr. Pêche. Piscic. 319 (1989) 146-168. 
[6] Chevassus B., Dupont-Nivet M., Mauger S., Haffray P., Vandeputte M., Estimation of heritabilities in two groups of rainbow trout (Onchorhynchus mykiss) with families mixed together since eyed stage, in: Proc. 7th World Cong. Genet. Appl. Livest. Prod., Montpellier, 19-23 August 2002, Inra, CastanetTolosan, France, CD-ROM communication 06-04.

[7] Chevassus B., Krieg F., Guyomard R., Blanc J.M., Quillet E., The genetics of brown trout: twenty years of French research, Buvisindi Icel. Agric. Sci. 6 (1992) 109-124.

[8] Donaldson L.R., Selective breeding of salmonoid fishes, in: McNeil W. (Ed.), Marine Aquaculture, Oregon State University Press, 1968, pp. 65-74.

[9] Elvingson P., Johansson K., Genetic and environmental components of variation in body traits of rainbow trout (Oncorhynchus mykiss) in relation to age, Aquaculture 118 (1993) 191-204.

[10] Falconer D.S., Introduction to quantitative genetics, Longman, New York, 1981.

[11] Fishback A.G., Danzmann R.G., Ferguson M.M., Gibson J.P., Estimates of genetic parameters and genotype by environment interactions for growth traits of the rainbow trout (Oncorhynchus mykiss) as inferred using molecular pedigrees, Aquaculture 206 (2002) 137-150.

[12] Friars G.W., Bailey J.K., Coombs K.A., Realized responses to selection in Atlantic salmon, in: Proc. 4th World Conf. Genet. Appl. Livest. Prod., 1990, Vol. 16, Edinburgh, Scotland, pp. 159-162.

[13] Gall G.A.E., Influence of size of eggs and age of female on hatchability and growth in rainbow trout, Calif. Fish Game 60 (1974) 26-35.

[14] Gelineau A., Corraze G., Boujard T., Effects of restricted ration, time-restricted access and reward level on voluntary food intake, growth and growth heterogeneity of rainbow trout (Oncorhynchus mykiss) fed on demand with self-feeders, Aquaculture 167 (1998) 247-258.

[15] Gjedrem T., Genetic variation in quantitative traits and selective breeding in fish and shellfish, Aquaculture 33 (1983) 51-72.

[16] Gjerde B., Growth and reproduction in fish and shellfish, Aquaculture 57 (1986) $37-55$.

[17] Gjerde B., Simianer H., Refstie T., Estimates of genetic and phenotypic parameters for body weight, growth rate and sexual maturity in Atlantic salmon, Livest. Prod. Sci. 38 (1994) 133-143.

[18] Henryon M., Jokumsen A., Berg P., Lund I., Pedersen P.B., Olesen N.J., Slierendrecht W.J., Genetic variation for growth rate, feed conversion efficiency, and disease resistance exists within a farmed population of rainbow trout, Aquaculture 209 (2002) 59-76.

[19] Hershberger W.K., Myers J.M., Iwamoto R.N., McCauley W.C., Saxton A.M., Genetic changes in the growth of coho salmon (Oncorhynchus kisutch) in marine-net pens, produced by ten years of selection, Aquaculture 85 (1990) 187-197.

[20] Huang C.M., Liao I.C., Response to mass selection for growth rate in Oreochromis niloticus, Aquaculture 85 (1990) 199-205. 
[21] Hulata G., Moav R., Wohlfarth G.W., The effects of maternal age, relative hatching time and density of stocking on growth rate of fry in the European and Chinese race of the common carp, J. Fish Biol. 9 (1976) 499-513.

[22] Kause A., Ritola O., Paananen T., Mantysaari E., Eskelinen U., Selection against early maturity in large rainbow trout Oncorhynchus mykiss: the quantitative genetics of sexual dimorphism and genotype-by-environment interactions, Aquaculture 228 (2003) 53-68.

[23] Krieg F., Guyomard R., Population genetics of French brown trout (Salmo trutta L.): large geographical differentiation of wild populations and high similarity of domesticated stocks, Génét. Sél. Évol. 17 (1985) 225-242.

[24] Mckay L.R., Friars G.W., Ihssen P., Genetic parameters of growth in rainbow trout, Salmo gairdneri, as a function of age and maturity, Aquaculture 58 (1986) 241-254.

[25] Mckay L.R., Friars G.W., Ihssen P., Genetic parameters of growth in rainbow trout, Salmo gairdneri, prior to maturation, Can. J. Genet. Cytol. 28 (1986) 306-312.

[26] Moav R., Wohlfarth G.W., Magnification through competition of genetic differences in yield capacity in carp, Heredity 33 (1974) 181-202.

[27] Moav R., Wohlfarth G.W., Two way selection for growth rate in the common carp (Cyprinus carpio L.), Genetics 82 (1976) 83-101.

[28] Nilsson J., Genetics of growth of juvenile Arctic char, Trans. Am. Fish. Soc. 123 (1994) 430-434.

[29] O'Flynn F.M., Bailey J.K., Friars G.W., Responses to two generations of index selection in Atlantic salmon (Salmo salar), Aquaculture 173 (1999) 143-148.

[30] Pante M.J.R., Gjerde B., McMillan I., Inbreeding levels in selected populations of rainbow trout, Oncorhynchus mykiss, Aquaculture 192 (2001) 213-224.

[31] Robinson B.W., Doyle R.W., Phenotypic correlations among behavior and growth variables in tilapia: implications for domestication selection, Aquaculture 85 (1990) 177-186.

[32] Ruzzante D.E., Domestication effects on aggressive and schooling behaviour in fish, Aquaculture 120 (1994) 1-24.

[33] Ruzzante D.E., Doyle R.W., Rapid behavioural changes in medaka (Oryzias latipes) caused by selection for competitive and noncompetitive growth, Evolution 45 (1991) 1936-1946.

[34] Ruzzante D.E., Doyle R.W., Evolution of social behavior in a resource-rich, structured environment: selection experiments with medaka (Oryzias latipes), Evolution 47 (1993) 456-470.

[35] Silverstein J.T., Hershberger W.K., Genetics of size and growth rate through sexual maturity in freshwater-reared coho salmon (Oncorhynchus kisutch), Theor. Appl. Genet. 90 (1995) 733-739.

[36] Su G.S., Liljedahl L.E., Gall G.A.E., Effects of inbreeding on growth and reproductive traits in rainbow trout (Oncorhynchus mykiss), Aquaculture 142 (1996) 139-148.

[37] Teichert-Coddington D.R., Smitherman R.O., Lack of response by Tilapia nilotica to mass selection for rapid early growth, Trans. Am. Fish. Soc. 117 (1988) 297-300. 
[38] Vandeputte M., Kocour M., Mauger S., Dupont-Nivet M., De Guerry D., Rodina M., Gela D., Vallod D., Chevassus B., Linhart O., Heritability estimates for growth-related traits using microsatellite parentage assignment in juvenile common carp (Cyprinus carpio L.), Aquaculture 235 (2004) 223-236.

[39] Vandeputte M., Quillet E., Chevassus B., Early development and survival in brown trout (Salmo trutta fario L.): indirect effects of selection for growth rate and estimation of genetic parameters, Aquaculture 204 (2002) 435-445.

[40] Winkelman A.M., Peterson R.G., Heritabilities, dominance variation, common environmental effects and genotype by environment interactions for weight and length in chinook salmon, Aquaculture 125 (1994) 17-30.

To access this journal online: www.edpsciences.org 